\title{
Single Variable Unconstrained Optimization Techniques Using Interval Analysis
}

\author{
G. Veeramalai ${ }^{1}$, R.J.Sundararaj ${ }^{2}$ \\ 1(Assistant Professor, Dept.of Mathematics, Annai Mathammal Sheela Engineering College, Namakkal-637 \\ 013,Tamilnadu, India) \\ 2(Assistant Professor, Dept.of Mathematics, Annai Mathammal Sheela Engineering College, Namakkal-637 \\ 013,Tamilnadu, India)
}

Abstract: In this paper, we discussed single variable unconstrained optimization techniques using Interval Analysis. The most of the unconstrained linear problems have been dealt with differential calculus methods. But, here non-linear unconstrained problems are solved using Newton's method by establishing Interval Analysis method. Establishing Interval Analysis method gives more accurate root even for higher order derivatives.

Key words: Interval analysis, Newton's method, Single variable, unconstrained optimization techniques.

\section{Introduction}

We need to be clear that optimization in economic simply refers to the actions by which individual economic agents do as well as they can. Thus consumers who seek to maximize utility are optimizing when they choose consumption bundles which yield the highest level of utility. Similarly firms who seek to maximize profit given production functions, prices of output and inputs are optimizing when they choose input levels which yield the highest level of profits.

The differentiable function $f(x)$ to be maximized is concave. The necessary and sufficient Condition for $x=x^{*}$ to be optimal (a global max) is $\frac{d f}{d x}=0 \quad$ at $x=x^{*}$ It is usually not very easy to solve the above equation analytically.

The one dimensional unconstrained problems to find a sequence of trial solutions that leads toward an optimal solution and using the signs of derivative to determine where to move positive derivative indicates that $x^{*}$ is greater than $x$; and vice versa.

We propose a new method for finding the roots using interval analysis. Interval Analysis is a means of representing uncertainty by replacing single (fixed-point) values with intervals. Interval analysis is applied to numerical methods that deal with optimization functions.

\section{Preliminaries}

2.1 Global optimization: Global optimization is a branch of applied mathematics and numerical analysis that deals with optimization of a function or a set of functions according to some criteria. Typically, a set of bound and more general constraints is also present, and the decision variables are optimized considering also the constraints.

\subsection{Interval Arithmetic:}

We recall a new type of arithmetic operations on interval numbers introduced in [12]: For $\tilde{x}=\left[x_{1}, x_{2}\right]$

, $\tilde{y}=\left[y_{1}, y_{2}\right]$ and for $* \in\{+,-, \times, \div\}$

We define $\tilde{x} * \tilde{y}=[(m(\tilde{x}) * m(\tilde{y})-k),(m(\tilde{x}) * m(\tilde{y})+k)]$

Where $k=\min [(m(\tilde{x}) * m(\tilde{y})-\alpha),(\beta-m(\tilde{x}) * m(\tilde{y}))]$

Where $\alpha$ and $\beta$ are the end points of the interval $\tilde{x} \circ \tilde{y}$ under the existing interval arithmetic.

In particular,

i. Addition:

$\tilde{x}+\tilde{y}=\left[x_{1}+x_{2}\right]+\left[y_{1}+y_{2}\right]$ 


$$
=\{m(\tilde{x})+m(\tilde{y})-k, m(\tilde{x})+m(\tilde{y})+k\} \text { Where } k=\left\{\frac{\left(y_{2}+x_{2}\right)-\left(y_{1}+x_{1}\right)}{2}\right\}
$$

ii. Subtraction:

$$
\begin{aligned}
\tilde{x}-\tilde{y} & =\left[x_{1}+x_{2}\right]-\left[y_{1}+y_{2}\right] \\
& =\{m(\tilde{x})-m(\tilde{y})-k, m(\tilde{x})-m(\tilde{y})+k\} \text { Where } k=\left\{\frac{\left(y_{2}+x_{2}\right)-\left(y_{1}+x_{1}\right)}{2}\right\}
\end{aligned}
$$

iii. Multiplication:

$$
\tilde{x} . \tilde{y}=\tilde{x} \tilde{y}=\left[\begin{array}{ll}
x_{1} & x_{2}
\end{array}\right]\left[\begin{array}{ll}
y_{1} & y_{2}
\end{array}\right]=\{m(\tilde{x}) m(\tilde{y})-k, m(\tilde{x}) m(\tilde{y})+k\}
$$

Where $k=\min [(m(\tilde{x}) m(\tilde{y})-\alpha),(\beta-m(\tilde{x}) m(\tilde{y}))]$

Where $\alpha=\min \left(x_{1} y_{1}, x_{1} y_{2}, x_{2} y_{1}, x_{2} y_{2}\right)$

Where $\beta=\max \left(x_{1} y_{1}, x_{1} y_{2}, x_{2} y_{1}, x_{2} y_{2}\right)$.

\section{iv. Inverse:}

$\tilde{x}^{-1}=\frac{1}{\tilde{x}}=\frac{1}{\left[x_{1}, x_{2}\right]}=\left[\frac{1}{m(\tilde{x})}-k, \frac{1}{m(\tilde{x})}+k\right]$

Where $k=\min \left\{\frac{1}{x_{2}}\left(\frac{x_{2}-x_{1}}{x_{1}+x_{2}}\right), \frac{1}{x_{1}}\left(\frac{x_{2}-x_{1}}{x_{1}+x_{2}}\right)\right\} \& \quad 0 \notin\left[x_{1}, x_{2}\right]$

From iii. It is clear that $\lambda \tilde{x}= \begin{cases}{\left[\lambda x_{1}, \lambda x_{2}\right]} & \text { for } \lambda \geq 0 \\ {\left[\lambda x_{2}, \lambda x_{1}\right]} & \text { for } \lambda<0\end{cases}$

It is to be noted that we use $\circ$ to denote the existing interval arithmetic and $*$ to denote the modified interval arithmetic. But wherever there is no confusion we use the same notation for both the cases.

It is also to be noted that $\tilde{x} \circ \tilde{y} \subseteq \tilde{x} * \tilde{y}\{x \circ y / x \subseteq \tilde{x}, y \subseteq \tilde{y}\}$, where $\circ \in\{\otimes, \oplus, \Theta, \mathrm{O}\}$ is the existing interval arithmetic.

\section{Single- Variable Theory}

Given a real valued function $f(x)$ defined on a subset $\mathrm{S}$ of $\mathrm{R}$, if $f$ attains its maximum (or minimum) at an interior point $x_{0} \in S$, then $f^{\prime}(x)=0$, provided $f$ is differentiable at $x_{0}$.Otherwise attains its maximum (or minimum) on the boundary of $\mathrm{S}$.

\subsection{Determining single variable Techniques}

Most of the Numerical methods in optimization techniques have characteristics for improved approximation values to the optimal solution, based on specific methods. The two methods we will discuss are Elimination and Interpolation methods. These methods are used to obtain values of the objective function. For the given function, maximum or minimum values can be obtained by having various combinations of the decision variables. Then conclusions are drawn regarding the optimal solutions.

\subsection{Elimination Method}

The elimination method can be used to find optimal solutions for even discontinuous functions. To use the elimination methods, however, the function must have only one maximum or minimum (ie, be external). Even if a function is not globally extrema, if it is extrema over some interval, the method may be applied over that interval. For elimination methods, an interval is the result. If we seek a single answer, then we evaluate the function at the end points and midpoint of the interval, and take the optimum of the three values to approximate the desired value. 


\subsection{Interpolation Method}

A method for approximation or precisely finding some quantity by known individual values of it or of other quantities related to it. On the basis of interpolation a whole series of approximate methods for solving mathematical problem has been developed

Most significant in numerical mathematics is the problem of constructing means for the interpolation of the functions. The interpolation of functions and operator is also widely used in constructing numerical methods.

\section{Newton's Method}

Newton's Method is an iterative method used to determine the root of a function. Given a function $f(x)$ and an initial guess to the root $x_{0}$, improved guesses are given by

$$
x_{i+1}=x_{i}-\frac{f\left(x_{i}\right)}{f^{\prime}\left(x_{i}\right)} i \geq 1
$$

In order to apply Newton's Method to find the critical points of a function, the function's first and second derivatives must exist in the neighborhood of interest. If the second derivative at an iterate $x_{i+1}$ is zero, the method is fails. Note that since Newton's Method is only a root-finding method, it can only determine where the first derivative is zero; It cannot determine if this is a maximum or minimum. The sign of the second derivative must be examined to determine this. Here is the idea: given an initial guess $x_{1}, x_{2}$ and two tolerances.

$\epsilon=$ the tolerance in the difference between successive iterate and

$\delta=$ the tolerances in the derivative of the function do the following: while $\left|f^{\prime}(x)\right| \geq \delta$ and $\left|x_{i}-x_{i-1}\right| \leq \varepsilon$

Compute $x_{i}=x_{i-1}-\frac{f\left(x_{i-1}\right)}{f^{\prime}\left(x_{i-1}\right)}$

\section{Single Variable Unconstrained Optimal Solutions}

Given a real valued function $\mathrm{f}(\mathrm{x})$ defined on $\mathrm{X}$ in $\mathrm{R}$, if $\mathrm{f}$ attains its maximum or minimum at a point $x_{0} \in X$, then the roots of $f^{\prime}(x)=0$ can be calculated by using Newton's interval method, which is substituted on ' $\mathrm{f}$ ' to obtain maximum or minimum value of ' $\mathrm{f}$ '.

\subsection{Interval Newton Method}

For details of the math and convergence properties of the algorithm, refer to Kulisch et al. (2001). This algorithm, when finding roots of fixed-point functions exhibits $\mathrm{O}\left(\mathrm{n}^{\wedge} 2\right)$ convergence. Like the Bisection Method, it requires a bracketing interval to begin and with each iteration generates smaller and smaller intervals (if possible), which are bounded by intersections with previous iterations. The algorithm is as follows:

$$
x_{i+1}=\left(m\left(x_{i}\right)-\frac{f\left(m\left(x_{i}\right)\right)}{f^{\prime}\left(x_{i}\right)}\right) \cap x_{i}
$$

Where the $\mathbf{x}$ 's are intervals, $\mathrm{m}(\mathbf{x})$ is the midpoint of a interval $\mathbf{x}$, and $\mathrm{f}$ is the function whose root we seek (Kulisch et al. 35-36). The similarity of this algorithm to the fixed-point Newton method is that a starting interval must be supplied and the interval size is decreased using a $f(\mathbf{x}) / \mathrm{f}^{\prime}(\mathbf{x})$ term during iterations. This algorithm is almost as simple as the bisection method since an easy convergence criterion has been specified; however, the interval Newton requires implementation of root func' $(t)$ and requires $0 \notin \operatorname{rootfunc}^{\prime}(x)$ for an evaluated interval $\mathbf{x}$. Through computational trials, I have decided to increase the complexity of the function evaluations for the $f(\mathbf{x}) / f^{\prime}(\mathbf{x})$ by computing its united extension instead of a interval extension. Since both $f(\mathbf{x})$ and $\mathbf{f}^{\prime}(\mathbf{x})$ involve many interval arithmetic calculations in an interval extension, the values are over expanded from their true solution set and in computing optimal residence times we are interested in finding tight bounds on the root given model uncertainties. As a result, the interval Newton step is calculated by finding the minimums and maximums of $f(\mathbf{x})$ and $f^{\prime}(\mathbf{x})$ given all the combinations of the endpoints of the parameters and the time interval and producing united extension values for both quantities.

\subsection{Example 1:}

For the funciton $f=x^{3}-6 x+3$, maximum value can be calculated as follows

Then $f^{\prime}=3 x^{2}-6=3\left(x^{2}-2\right)$

To find the value of $f^{\prime}=3 x^{2}-6$, using Newton's interval methods 
Let us consider $F=x^{2}-2$

An interval expansion of $F^{\prime}=2 x$ is $F^{\prime}(X)=2 X$

Hence $N(X)=m(X)-\frac{[m(X)]^{2}-2}{2 X}$

Newton's interval formula $X^{(k+1)}=X^{(k)} \cap m(X)-\frac{[m(X)]^{2}-2}{2 X}$

Taking $X^{(0)}=[1,2]$, we get $X^{(1)}=\left[\frac{22}{16}, \frac{22}{16}\right], X^{(2)}=[1.41406,1.41414]$ and

$X^{(3)}=[1.41421335,1.4142135]$ of course $F=x^{2}-2$ has solution $x=\sqrt{2}$ rounding $X^{(3)}$ out of the eight place. We see that $\sqrt{2}$ lies on $[1.41421335,1.4142135]$

(i.e) $X^{*}=[1.41421335,1.4142135]$ to be optimal value of $\mathrm{F}$, then $X^{*}$ using in given equation. We obtain the maximum value -2.36139 .

\section{Example 2:}

The following function $f(x)=\frac{1}{3}\left(4567 x^{3}-13701 x^{2}+13701 x+13701\right)$ has also maximum value as derived below.

Then $f^{\prime}(x)=4567 x^{2}-9134 x+4567$

To find the value of $f^{\prime}(x)=4567 x^{2}-9134 x+4567$, using Newton's interval methods

Let us consider $F=4567 x^{2}-9134 x+4567$

An interval expansion of $F^{\prime}=9134 x-9134$ is $F^{\prime}(X)=9134 X-9134$ and $F^{\prime \prime}(X)>0$

Hence $N(X)=m(X)-\frac{9134[m(X)]-9134}{9134}$

Newton's interval formula $X^{(k+1)}=X^{(k)} \cap m(X)-\frac{9134[m(X)]-9134}{9134}$

Taking $X^{(0)}=[0,3]$, we get $X^{(1)}=[0.7585,0.8909], X^{(2)}=[0.9349,1.088], X^{(3)}=[1.036,1.041]$, $X^{(4)}=[0.9298,1.024], X^{(5)}=[0.9856,1.024]$ and $X^{(6)}=[0.9852,1.015]$

using in given function

$f([0.9852,1.015])=\frac{1}{3}\left(4567[0.9852,1.015]^{3}-13701[0.9852,1.015]^{2}+13701[0.9852,1.015]+13701\right)$

We obtain the maximum value 6089.3333.

\subsection{Real time Example:}

For real time problems also, Interval analysis method can be calculated to get optimal value. The following example describes the fact that how an optimal value obtained for the real time problem.

An animal weighing $200 \mathrm{~kg}$ gains $5 \mathrm{~kg}$ per day and costs Rs 0.60 per day to keep. The market price for animals is Rs 0.70 per day, but is falling Rs.0.01 Per day. When the animal be sold.

We define a mathematical modeling our problem is to maximize $\mathrm{p}(\mathrm{t})$ over the set $\{t ; t \geq 0\}$

Where $P(t)=140+0.09 t-0.05 t^{2}$

Find the maximum value of $P(t)=140+0.09 t-0.05 t^{2}$.

Then $\frac{d p}{d t}=0.9-2(0.05) t=0.9-0.10 t$

To find the value of $P^{\prime}=0.9-2(0.05) t=0.9-0.10 t$ using Newton's interval methods

Let us consider $F=0.9-0.10 t$ 
An interval expansion of $F^{\prime}=0.10$ is $F^{\prime}(X)=0.1$

Hence $N(X)=m(X)-\frac{0.9-0.1[m(X)]}{0.1}$

Newton's interval formula $X^{(k+1)}=X^{(k)} \cap m(X)-\frac{0.9-0.1[m(X)]}{0.1}$

Taking $X^{(0)}=[8,10]$, we get $X^{(7)}=[8.992,9.0012]$

Take an interval $t_{o}=[8.992,9.0012]$ using in $P(t)=140+0.09 t-0.05 t^{2}$,

We get a solution $P(t)=140+0.09[8.992,9.0012]-0.05[8.992,9.0012]^{2}$

$=144.04964$

The maximum value of $\mathrm{P}(\mathrm{t})$ is 144.04964 .

\section{CONCLUSION}

By calculus methods of calculating maxima and minima for linear derivatives gives approximated value. Thus, while using Interval Analysis method, calculating maxima and minima even for higher order derivatives is not tedious and it gives more accurate value than any other method. Also, this Interval Analysis method is very useful and economic in time saving one for most of the Engineering fields like in computing programmes, applied thermo dynamics etc.

\section{References:}

[1] E. Hansan and G. W. Walster, "Global optimization using Interval Analysis”, Marcel Dekker, New York, 2003.

[2] Karl Nickel, On the Newton method in Interval Analysis. Technical report 1136, Mathematical Research Center, University of Wisconsion, Dec1971

[3] Helmut Ratschek and Jon G. Rlkne, New Computer Methods for Global Optimization, Wiley, New York, 1988.

[4] Louis B. Rall, A Theory of interval iteration, proceeding of the American Mathematics Society, 86z:625-631, 1982.

[5] Louis B. Rall, Application of interval integration to the solution of integral equations. Journal of Integral equations 6: 127$141,1984$.

[6] Ramon E. Moore, R. Baker Kearfoth, Michael J. Cloud, Introduction to interval analysis, SIAM, 105-127, Philadelphia, 2009.

[7] Eldon Hansen, Global optimization using interval analysis- Marcel Dekker, 1992

[8] Hansen E.R (1978a), “Interval forms of Newton's method, Computing 20, 153-163.

[9] Hansen E. R (1979), "Global optimization using interval analysis-the one dimensional case, J.Optim, Theory Application, 29, 314331.

[10] Hansen E. R (1988), An overview of Global Optimization using interval analysis in Moore(1988) pp 289-307.

[11] K. Ganesan and P. Veeramani, On Arithmetic Operations of Interval Numbers, International Journal of Uncertainty, Fuzzines s and Knowledge - Based Systems, 13 (6) (2005), 619 - 631.

[12] K. Ganesan, On Some Properties of Interval Matrices, International Journal of Computational and Mathematical Sciences, 1 (2) (2007), 92-99.

[13] S. M. Guu, H. H. Chen, C. T. Pang, Convergence of products of fuzzy matrices, Fuzzy Sets Systems, 121 (2001) $203-207$.

[14] E. R. Hansen and R. R. Smith, Interval arithmetic in matrix computations, Part 2, SIAM. journal of Numerical Analysis, 4 (1967), $1-9$.

[15] E. R. Hansen, On the solution of linear algebraic equations with interval coefficients, Linear Algebra Appl, 2 (1969), 153 - 165 .

[16] E. R. Hansen, Bounding the solution of interval linear Equations, SIAM Journal of Numerical Analysis, 29 (5) (1992), 1493 1503.

[17] P. Kahl, V. Kreinovich, A. Lakeyev and J. Rohn, Computational complexity and feasibility of data processing and interval computations Kluwer Academic Publishers, Dordrecht (1998)

[18] E. Kaucher, Interval tler, A Fourth-Order Finite-Difference Approximation for the Fixed Membrane Eigen- problem, Math. Comp., 25 (1971),237 - 256.

[19] R. E. Moore, Automatic error analysis in digital computation, Technical Report LMSD4882, Lockheed Missiles and Space Division Sunnyvale,California, 1995.

[20] S. Ning and R. B. Kearfott, A comparison of some methods for solving linear interval Equations, SIAM Journal of Numerical Analysis, 34 (1997),1289 - 1305.

[21] J. Rohn, Inverse interval matrix, SIAM Journal of Numerical Analysis, 3(1993), 864 - 870.

[22] T.Nirmala, K. Ganesan, D.Datta, H.S.Kushwaha, Inverse interval matrix: A new approach, International Journals of Applied mathematical Science,1 (2) (2007), 92-99.

[23] R. E. Moore, Methods and Applications of Interval Analysis, SIAM, Philadelphia, 1979.

[24] A. Neumaier, Interval Methods for Systems of Equations, Cambridge University Press, Cambridge, 1990.

[25] G. Alefeld and J. Herzberger, "Introduction to Interval Computations",Academic Press, New York, 1983.

[26] E. R. Hansen and R. R. Smith, "Interval arithmetic in matrix computations", Part 2, SI AM. Journal of Numerical Analysis, vol. 4, pp. $1-9,1967$.

[27] E. R. Hansen, "Global Optimization Using Interval Analysis”, Marcel Dekker, Inc., New York, 1992.

[28] A. Neumaier, "Interval Methods for Systems of Equations", Cambridge University Press, Cambridge, 1990.

[29] J. Rohn, "Interval matrices: singularity and real eigenvalues", SIAM.Journal of Matrix Analysis and applications, vol. 1, pp. 82 91, 1993.

[30] S. Ning and R. B. Kearfott, "A comparison of some methods for solving linear interval Equations”, SIAM. Journal of Numerical Analysis, vol.34, pp. $1289-1305,1997$.

[31] E. R. Hansen and R. R. Smith, "Interval arithmetic in matrix computations”, Part 2, SI AM. Journal of Numerical Analysis, vol. 4, pp. $1-9,1967$. 